\title{
Design and Implementation of Medical Image Enhancement System Based on MATLAB GUI
}

\author{
Wen $\mathrm{He}$ \\ Chengdu medical college, Chengdu, Sichuan, 610500, China \\ hewen41731@163.com
}

Keywords: MATLAB GUI; Medical Image; Enhancement; System

\begin{abstract}
For the medical image of poor visual effect, we designed and implemented a medical image enhancement system. The system is based on MATLAB GUI development, and has the modules of file operations, image editing, image analysis, spatial domain enhancement, frequency domain enhancement and wavelet domain enhancement etc. It can realize the medical image editing, analysis, filtering, enhancement and denoising and other functions. This paper describes the overall structure and design method of the system, from the spatial domain, frequency domain and wavelet domain introduced the GUI implementation process of three kinds of enhancement algorithms.
\end{abstract}

\section{Introduction}

The Overall Design of the System. Medical image( $X$ ray, CT, MR, PET, ultrasound, etc.) is an important means of medical diagnosis, is an indispensable part of modern medicine, the image quality is good or bad has a great influence on the accuracy of medical diagnosis and treatment. Thus, it has increasingly high status in the clinical, not only in disease diagnosis has a large number of applications, still in surgery and radiation therapy planning and design, implementation plan and evaluating the curative effect also play an important role. Medical image has been able to become an important diagnostic means, mainly because of different organizational structures can have a good distinction to allow doctors to observe the different boundary on the image. However, due to the imaging device itself and the access conditions and other factors, may make the imaging performance of the original medical image is not very satisfactory, the extraction of the lesion site information is not well demonstrated, in which it is possible to image quality degradation occurs, some of the boundary structure is not obvious or obscure, some impact on the doctor's diagnosis, therefore, likely to cause doctors misdiagnosed. Then we can through the medical image processing technology for proper treatment, so that the boundary of the medical image is enhanced, highlight the image details, all kinds of organizational structure to separate, so it can more accurately reflect the lesions site and to make it easier for doctors to make a correct diagnosis, in order to reduce misdiagnosis and missed diagnosis of probability.

MATLAB GUI (MATLAB Graphical User Interface) is the interface information from the MATLAB graphic objects created for human-computer interaction. In MATLAB GUI, the users can put the program they want to run as well as various commands placed in the graphical user interface in the graphics window, menu, text boxes, buttons, and other control objects below. Through the mouse and keyboard and other simple input can achieve the results you want the program execution, so that you can avoid to writing a lot of complex commands in every execution, so as to achieve a simple human-computer information exchange.

In recent years, scholars have proposed many MATLAB GUI-based image processing system [1-5], but most of these systems for the general image or special images processing, for medical image processing system is not much. This paper according to the medical images features, designed and implemented a medical image processing system based on MATLAB GUI, which has the characteristics of friendly interface, feature-rich, simple operation and so on.

This paper designed and developed a medical image enhancement processing system based on MATLAB GUI, it can realize the medical image editing, analysis, filtering, enhancement and denoising and other functions. These modules were designed from the relevant enhancement 
algorithms in spatial domain, frequency domain and wavelet domain. Some classical image enhancement algorithms are classified according to the transform domain, easy for function additions and maintenance. The main function modules of the system are shown in Fig. 1.

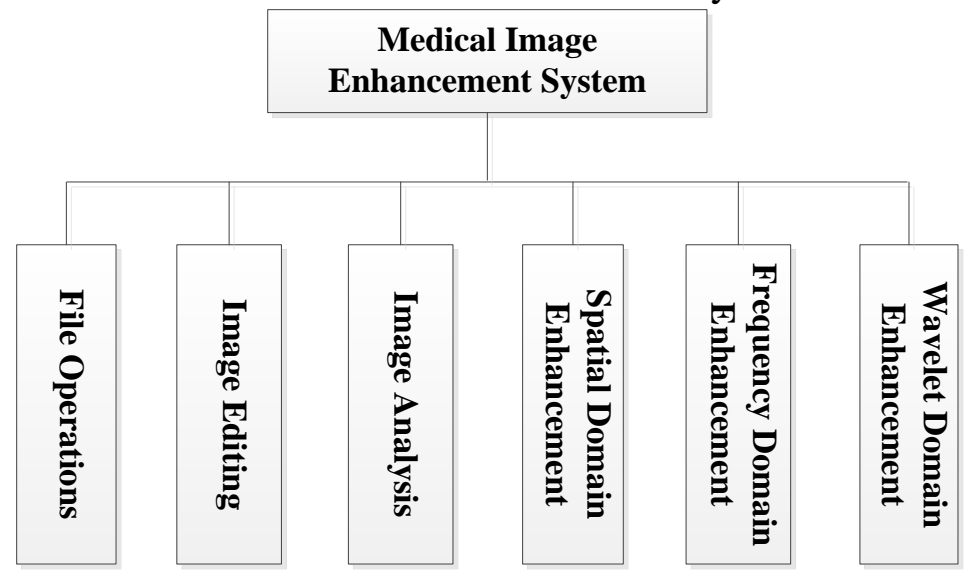

Figure 1. The main function modules of the system

The main design steps of the system are as follows:

(1).Create the main interface of the system, design the appearance and layout of the interface, create the main menu bar and submenus.

(2).Design GUI interface for each sub menu, add the required controls, set its properties.

(3).Write the callback functions of the submenus and controls, run to generate Fig files.

(4).Commissioning and operating the system, and gradually improve the system functions.

(5).Convert the system to an EXE files that can be run independently, and to publish.

\section{Design of the System Modules}

The system consists of mainly six functional modules, which contains file operations, image editing, image analysis, spatial domain enhancement, frequency domain enhancement and wavelet domain enhancement. The detailed designs of each functional module are as follows:

File Operations. File Operations module mainly to achieve the image open, preview, format conversion, save and exit function, you can select an image from the computer to display, convert its format, save the image of format conversion, and exit the system function.

Image Editing. Image Editing module mainly to achieve the image zooming, rotation, cropping, adjust brightness and contrast functions, the original medical image can be preprocessed, cut out the portion of interest.

Image Analysis. Image analysis module mainly includes pixel value display, image intensity description, image histogram and image contour map, etc., it can display the pixel value of any location of the image, display intensity description between any two points, display image histogram and image contour map.

Spatial Domain Enhancement. Spatial domain enhancement includes some commonly used algorithms of image spatial domain processing, including logarithmic transformation, exponential transform, piecewise linear transformation, histogram processing, Laplacian sharpening, mean filtering, median filtering, Wiener filtering and unsharp masking etc. The following unsharp masking algorithm as an example to introduce the GUI implementation process of the spatial domain enhancement algorithm.

The idea of the unsharp masking algorithm is firstly perform low-pass filtering on the original image to produce a passivation blurred image, and then the original image and blurred image subtraction to get the image which preserved the high frequency components, and then the high frequency image using a parameter to amplify and then superimposed on the original image, which produces an edge enhanced image. The realization process of the algorithm is as follows:

(1) Perform low-pass filtering on the original image $f(x, y)$ to get the blurred image $\bar{f}(x, y)$.

(2) Calculating the difference between the original image and the blurred image, and get the high 
frequency image, that is:

$$
g_{\text {mask }}(x, y)=f(x, y)-\bar{f}(x, y) .
$$

(3) The high-frequency image $g_{\text {mask }}(x, y)$ is multiplied by a weighting factor $k$ and then superimposed on the original image to obtain the enhanced images, that is:

$$
g(x, y)=f(x, y)+k^{*} g_{\text {mask }}(x, y) .
$$

The GUI implementation process is as follows:

(1) Design program interface, add Axes, Push Button, Slider, Edit Text, Static Text and other controls, as shown in Fig. 3.1.

(2) Write the callback function for each button, in which the function of the first button to open the image, the second button function to perform unsharp masking algorithm, the third button function for saving enhanced image, the fourth button to exit the function module. The callback function of the button "Open Image" is as follows:

[filename, pathname]= uigetfile (\{'*.jpg';'*.bmp';'*.gif';'*.tif';... $\},$ 'select image');

$\mathrm{f}=$ [pathname, filename];

$\operatorname{img}=$ imread $(f)$;

axes (handles.axes1);

imshow (img);

(3) Write the callback functions of Slider and Edit Text, by using the global variable, the value of the Slider or Edit Text is passed to the callback function of the processing button, so that the program can be set by the Slider or the Edit Text to set the algorithm's enhancement coefficient $k$ and the size of the passivation template. Wherein the callback function of the Slider which sets the enhancement coefficient of the algorithm is written as follows:

global k;

k=get (handles.slider1,'value');

set (handles.edit1,'string',k);

(4) Debugging and running the program. The Fig. 2 for the $15 \times 15$ passivation template, the enhancement factor $k$ is set to 10 for the processing results of the unsharp masking.

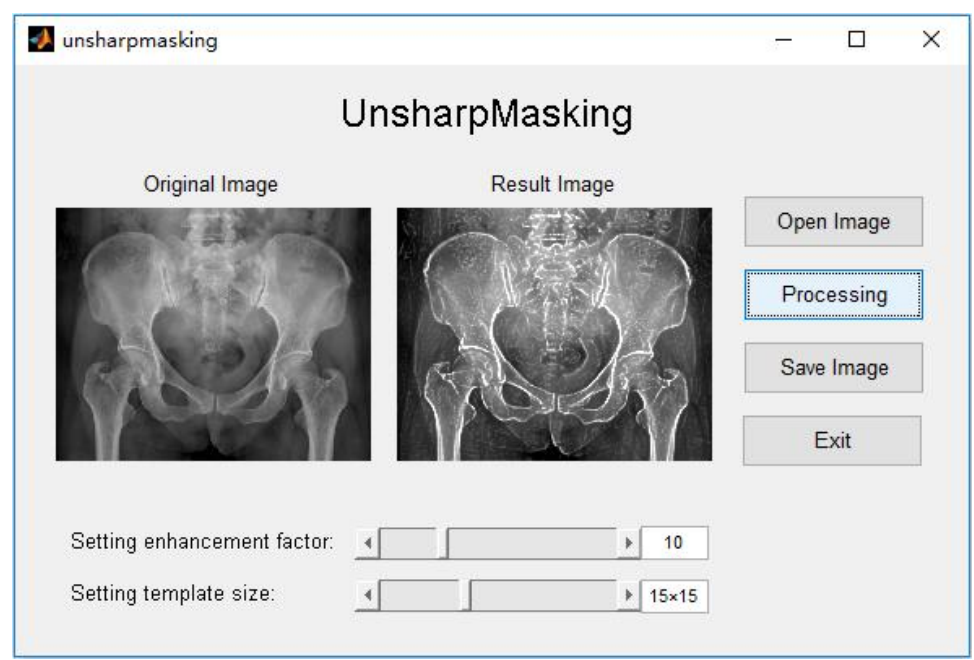

Figure 2. GUI design of unsharp masking 


\section{Frequency Domain Enhancement}

Frequency domain enhancement module contains some commonly used algorithms in frequency domain, including low-pass filtering, high-pass filtering, high frequency emphasis filtering and homomorphic filtering, etc. This paper takes the homomorphic filtering as an example, introduces the implementation process of the frequency domain enhancement algorithm.

Homomorphic filtering is an image processing method for the frequency filtering and grayscale transformation combine; it relies on the image illumination/reflectance model as the basis for frequency domain processing, by compressing the range of the brightness and enhancing the contrast to improve the image quality. Using this method can make the image processing in line with the human eye for the brightness response of the nonlinear characteristics, to avoid direct Fourier transform processing for image distortion. The implementation steps of the algorithm are as follows:

(1) According to the illumination/reflectance model [6], the original image is expressed as the product of the illumination function $f_{i}(x, y)$ and the reflection function $f_{r}(x, y)$ :

$$
f(x, y)=f_{i}(x, y) f_{r}(x, y) \text {. }
$$

(2) Since the Fourier transform of product is indivisible, so on both sides of the Eq. 3 to take the logarithm, get:

$$
\ln f(x, y)=\ln f_{i}(x, y)+\ln f_{r}(x, y) .
$$

(3) Let $z(u, v)=\ln (x, y)$, then the Fourier transform of the Eq. 4 to give:

$$
\mathfrak{I}\{z(u, v)\}=\mathfrak{I}\{\ln f(x, y)\}=\mathfrak{I}\left\{\ln f_{i}(x, y)\right\}+\mathfrak{I}\left\{\ln f_{r}(x, y)\right\} .
$$

(4) I.e. $Z(u, v)=F_{i}(u, v)+F_{r}(u, v)$, where $F_{i}(u, v)$ and $F_{r}(u, v)$ are the Fourier transforms of the $\ln f_{i}(x, y)$ and $\ln f_{r}(x, y)$.

(5) Select a homomorphic filter $H(u, v)$ to filter the $Z(u, v)$, the filter function of the system is selected as: $H(u, v)=(r H-r L) /\left(1+c D_{0} / D(u, v)\right)^{2 n}+r L$. Wherein $r H$ represents the high-frequency gain, $r L$ represents the low-frequency gain, $D_{0}$ as the cut-off frequency, $c$ is a positive constant.

(6) Convert the filtered $Z(u, v)$ back to the spatial domain, and then take its index can be obtained the enhanced image.

The flow of the algorithm is shown in the Fig. 3

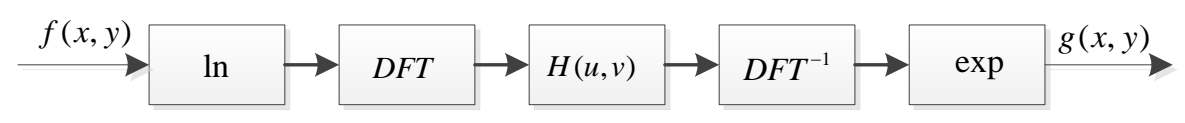

Figure 3. Algorithm flow chart

The Fig. 4 is the result of the homomorphic filtering with the filter parameters were set to $D_{0}=30, r H=1, r L=0.2$.

\section{Wavelet Domain Enhancement}

This module mainly includes wavelet enhancement, wavelet passivation, wavelet sharpening and wavelet denoising and other functions. Wavelet analysis is a popular technology, due to the multi-resolution wavelet analysis can effectively suppress noise, enhance the interest portion of the image, thus wavelet transform has been widely used in image processing. In this paper, we take the wavelet denoising as an example to introduce the implementation of wavelet de-noising module. 


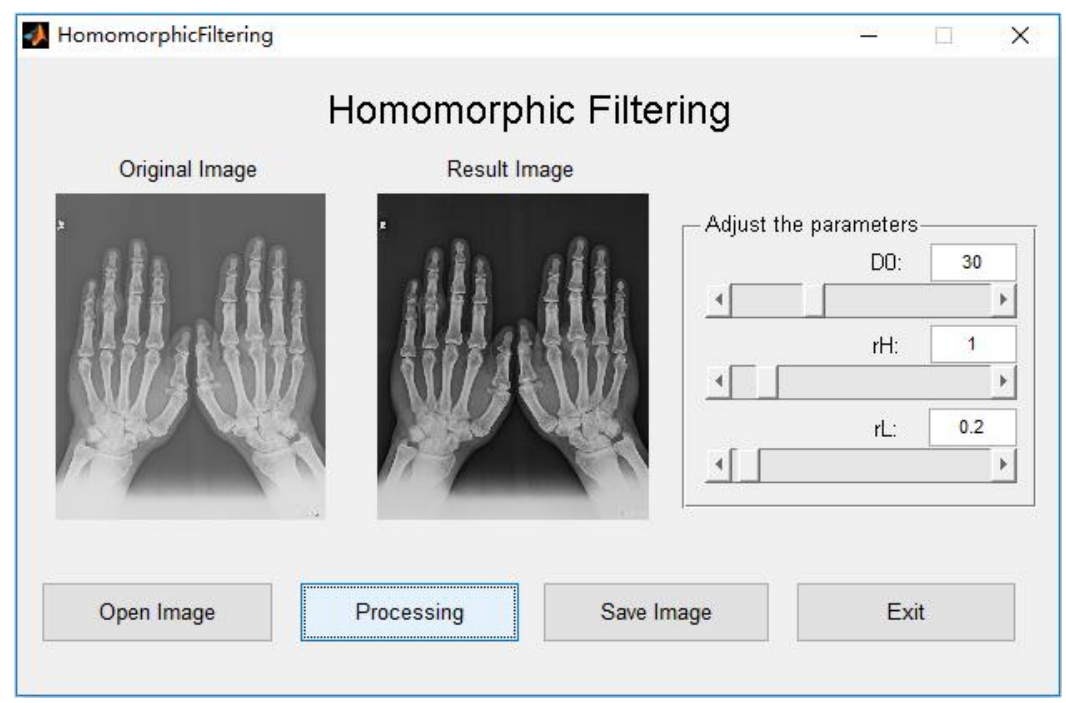

Figure 4. GUI design of Homomorphic Filtering

Wavelet transform decompose an image into each scale of the components of low-frequency, horizontal high-frequency, vertical high-frequency and the diagonal high-frequency. After the transformation, according to the need of image processing, some components of different position and different directions can be changed, so that the components of interested are amplified and the unneeded components are decreased. In practical application, for images with noise, after the wavelet decomposition, due to the image and the noise after wavelet transform with different statistical properties, the energy of the image itself corresponding to large amplitude of wavelet coefficients, mainly concentrated in low-frequency; the energy of noise corresponding to small amplitude of the wavelet coefficients, mainly concentrated in high-frequency, and distribution in each layer of the coefficients after wavelet transform [7]. According to this feature, we can set a threshold value to retain the wavelet coefficients which greater than the threshold and remove the wavelet coefficients which less than the threshold, to achieve the purpose of denoising.

The implementation steps of the algorithm are as follows:

(1) Decompose the image by wavelet transform: Select a wavelets and wavelet decomposition level $\mathrm{N}$ and then calculate the signal $\mathrm{S}$ to the $\mathrm{N}$-th layer decomposition.

(2) Threshold quantization for the high-frequency coefficients: for each layer of the 1 to $\mathrm{N}$ signal of the wavelet decomposition, select a threshold to shrinkage the high frequency coefficients of this layer. The selection of threshold is mainly has hard-threshold and soft-threshold two approaches [8, 9], the expression of the hard-threshold function is:

$$
w_{t}=\left\{\begin{array}{l}
w,|w| \geq t \\
0,|w|<t
\end{array}\right.
$$

The expression of the soft-threshold function is:

$$
w_{t}=\left\{\begin{array}{l}
\operatorname{sign}(w)(|w|-t),|w| \geq t \\
0, \quad|w|<t
\end{array}\right.
$$

Where $w$ is the wavelet coefficient, $t$ is the threshold value, and $w_{t}$ is the size of the wavelet coefficients after applying the threshold value.

The treatment of the hard-threshold method is: when the absolute value of the wavelet coefficients greater than the threshold, keep it unchanged; when the absolute value of the wavelet coefficients less than the threshold, set it to zero. While the soft-threshold method is when the absolute value of the wavelet coefficients greater than the threshold, let it all subtract the threshold; when the absolute value of the wavelet coefficients less than the threshold, set it to zero. 
Hard-thresholding method can better preserve the image edge and local feature, but get the estimated wavelet coefficients continuity was poor; while the continuity of the estimated wavelet coefficients obtained by soft-threshold is better, with a higher signal to noise ratio, but the treatment process is relatively smooth, it may cause blurred edges and other distortions [10]. In practical applications, it can be selected according to the processing needs.

3) 2-D Wavelet Reconstruction: computing the wavelet reconstruction of two-dimensional signal according to the $\mathrm{N}$-th layer of the low-frequency coefficients and the first layer to the N-th layer of the modified high-frequency coefficients.

The Fig. 5 is the GUI of the wavelet denoising module, which can realize the reading of the original image, display, noise adding and denoising function. In the parameter setting, we can set the type and the probability density of noise, wavelet and wavelet decomposition level, denoising method and so on. As shown below, the four Axes on the interface shows the processing results of wavelet soft threshold denoising after adding noise to an ultrasonic image.

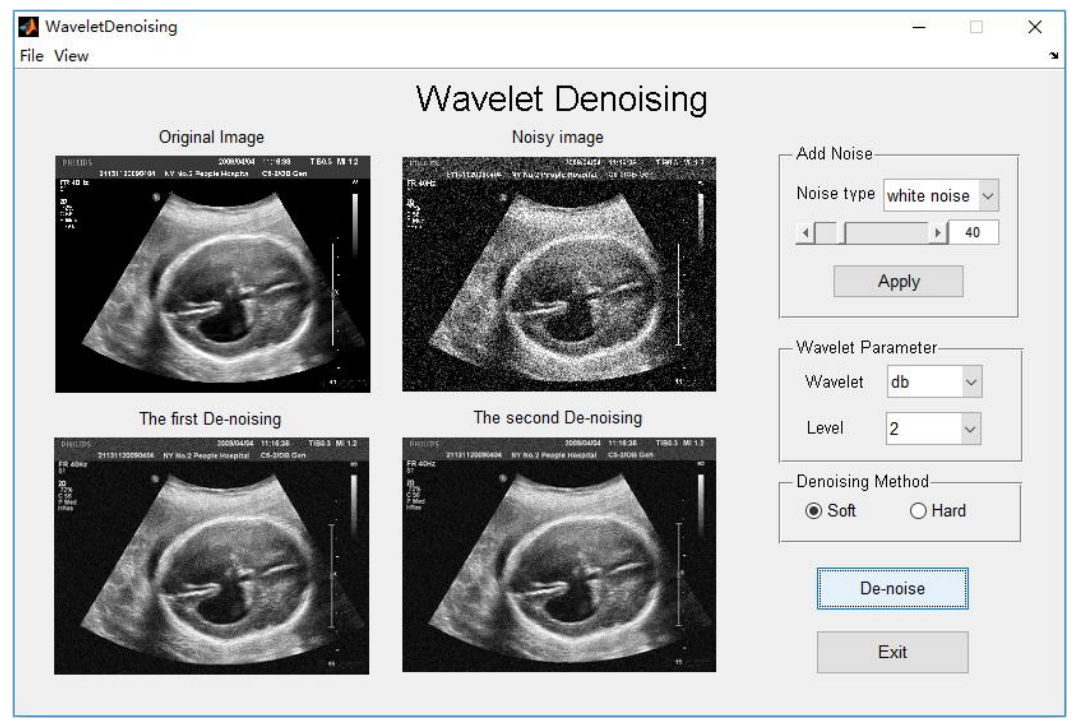

Figure 5. GUI design of Wavelet Denoising

\section{Summary}

In this paper, we designed and implemented a set of medical image enhancement processing system, it can realize the medical image editing, analysis, filtering, enhancement and denoising and other functions. The system has friendly interface,simple operation. From the spatial domain, frequency domain and wavelet domain classification design image enhancement modules, with good maintainability and scalability. With the continuous development of medical image processing technology, such systems will be more and more applications.

\section{Acknowledgement}

This project was supported by Chengdu Medical College Science Project (No. CYZ11-018) and Sichuan Education Department Science Project (No. 16ZB0283).

\section{References}

[1] N. Yao and Q. Geng: Computer engineering \& Software, Vol. 37 (2016). No.3, p.44. (In Chinese)

[2] B.F. Li and Y.Q. Xing: Computer \& Digital Engineering, Vol. 42 (2014). No.11, p.2177. (In Chinese) 
[3] H. Lan, J. Tian, S.Z. Li and L.H. Liu: Journal of Jiangxi University of Science and Technology, Vol. 35 (2014). No.3, p.79. (In Chinese)

[4] Y.Z. Guo and P.P. Jiao: Computer Technology and Development, Vol. 23 (2013). No.7, p.230. (In Chinese)

[5] Y. Xue: Electronic Design Engineering, Vol. 22 (2014). No.15, p.101. (In Chinese)

[6] Rafael C.Gonzalez and Richard E.Woods: Digital Image Processing, Third Edition(Publishing House of Electronics Industry, China 2013), p.182.

[7] M. Guo, Y.L. Ma and T. Zhu: Chinese Journal of Medical Imaging Technology, Vol.22 (2006) No.9, p.1435. (In Chinese)

[8] D.L. Donoho: IEEE Transactions on Information Theory, Vol.41 (1995) No.3, p.613.

[9] D.L. Donoho and I.M. Johnstone: Biometrika, Vol. 81 (1994) No.3, p.425.

[10]C. Zeng: the Research of Image De-noising and Enhancement Based on Wavelet Theory (MS., Wuhan University of Technology, China 2008), p.23. 